\title{
Widely tunable long-period waveguide grating filters
}

\author{
Kin Seng Chiang ${ }^{\mathrm{a}}$ \\ Department of Electronic Engineering, City University of Hong Kong, 83 Tat Chee Avenue, \\ Kowloon, Hong Kong, China
}

\begin{abstract}
This paper presents a review of the development of long-period waveguide gratings (LPWGs) with focus on the realization of various kinds of tunable devices, such as band-rejection filters, variable attenuators, band-pass filters, and add/drop multiplexers. A number of specific examples are given to demonstrate how the geometry and material flexibility of the optical waveguide technology facilitates the design of widely tunable LPWG-based devices. LPWG is evolving into a versatile waveguide structure for the implementation of a wide range of optical functions.
\end{abstract}

Keywords: Long-period gratings, optical devices, optical filters, optical waveguides, polymer waveguides, waveguide gratings.

\section{INTRODUCTION}

The first demonstration of a fiber grating in a single-mode fiber to achieve light coupling from the guided mode to the cladding modes of the fiber [1] has led to a simple technology for the realization of optical fiber filters. The pitch of the grating required is of the order of $100 \mu \mathrm{m}$, which is more than two orders of magnitude larger than that of the wellknown fiber Bragg grating (FBG), and, therefore, the grating is widely referred to as the long-period fiber grating (LPFG). The transmission spectrum of a typical LPFG consists of a number of rejection bands at specific wavelengths, which correspond to the couplings to various cladding modes of the fiber. A LPFG can be in the form of a periodic refractive-index modulation (produced by UV irradiation, femosecond-pulse irradiation, $\mathrm{CO}_{2}$-laser irradiation, etc.) or physical deformation along the fiber. LPFGs offer many nice features, including easy fabrication, low insertion loss, and negligible back reflection. Because of the nature of the grating, LPFG-based devices are usually broadband devices. Over the last ten years, there have been numerous developments in the application of LPFGs in fiber communication and sensing (see the review papers $[2,3]$ and the references therein).

Of the many applications of LPFGs, the realization of tunable filters is of particular interest in fiber communication systems as such filters can potentially offer active control in optical networks. Unfortunately, the resonance wavelength of an ordinary LPFG written in a conventional communication fiber shifts by only 3 to $10 \mathrm{~nm}$ for a change of $100{ }^{\circ} \mathrm{C}$ in temperature, which is not sufficient for many applications. Several techniques have been demonstrated for enhancing the wavelength tunability of LPFGs. By carefully choosing the order of the cladding mode and the operating wavelength, it is possible to increase the sensitivity of the resonance wavelength to a number of physical parameters (e.g., temperature, strain, and the surrounding refractive index). For example, a temperature sensitivity of $275 \mathrm{~nm} / 100{ }^{\circ} \mathrm{C}$ has been obtained with a LPFG written in a photosensitive B-Ge co-doped fiber by using this technique [4]. The wavelength tuning range of the grating, however, is rather limited (less than $30 \mathrm{~nm}$ ) [4]. The temperature sensitivity of a LPFG can also be increased by coating the grating with a polymer material that has a large thermal-optic coefficient. By using a UVcurable acrylate-based polymer coating, a temperature sensitivity of $80 \mathrm{~nm} / 100{ }^{\circ} \mathrm{C}$ has been achieved [5]. The sensitivity can be further enhanced by writing a grating in a microstructure fiber with large holes in the cladding filled with polymer [5]. Another way to increase the tunability of a LPFG is by etching of the fiber cladding. It is found that the resonance wavelength of a LPFG becomes much more sensitive to the surrounding refractive index after cladding etching [6]. A wide tuning range is thus possible by changing the surrounding refractive index.

The difficulty of enhancing the tunability of a LPFG is very much due to the geometry and material constraints of the optical fiber. To relax the constraints of the optical fiber, long-period gratings (LPGs) formed in planar waveguides,

${ }^{a}$ E-mail: eeksc@ cityu.edu.hk; Tel: (852) 2788 9605; Fax: (852) 27887791 
referred to as long-period waveguide gratings (LPWGs), have been proposed [7,8]. The flexibility of the optical waveguide technology allows LPGs to be fabricated in different waveguide structures with different materials. With the waveguide technology, it is no longer necessary to rely on a polymer coating to increase the temperature sensitivity of a LPG; the LPG can simply be formed in a polymer waveguide and ultra-high temperature sensitivity is almost guaranteed [9-15]. Furthermore, it is found that the characteristics of a LPWG depends critically on the physical dimensions of the waveguide [8,16]. A precise control of the dimensions of the waveguide, as provided by the modern microfabrication technology based on various lithographic techniques, can be used effectively to tailor the wavelength tunability of the grating. For example, the temperature sensitivity of a LPWG can be changed over a wide range by simply controlling the cladding [12,13] or the core thickness [14] of the waveguide, depending on the waveguide structure. Compared with LPFGs, LPWGs offer much higher flexibility in the design of optical devices, especially active devices. LPWGs can also serve as building blocks in integrated optical circuits.

LPWGs have been demonstrated experimentally with different materials, including glass $[10,12,17]$, polymer $[9-15,18$ 28], and semiconductor [29], and in different waveguide structures, including slab waveguides [9,10,12], ridge waveguides [13,15], rib waveguides [22], and channel waveguides [11,14,18-21,23-29]. The large thermo-optic coefficient of polymer (about 25 times larger than that of glass) allows the rejection bands of many of the polymer LPWGs to be tuned thermally over a wide range of wavelengths [9-15,24-26]. For example, the first demonstrated widely tunable LPWG [10], which is fabricated in a polymer-clad ion-exchanged glass waveguide, provides linear wavelength tuning over the entire $\left(\mathrm{C}+\mathrm{L}\right.$ )-band (from 1520 to $1610 \mathrm{~nm}$ ) with a temperature control of only $\sim 10{ }^{\circ} \mathrm{C}$, which corresponds to a temperature sensitivity of $\sim 9 \mathrm{~nm} /{ }^{\circ} \mathrm{C}$. This LPWG is superior to the LPFG tunable filters [4,5] in both the tuning range and the sensitivity. The large thermo-optic coefficient of polymer has also been exploited for the realization of LPWGs with a tunable contrast at the resonance wavelength [19,20,23,26,27,30,31]. A metal LPWG deposited on a properly designed polymer waveguide can in fact offer both a widely tunable center wavelength and a widely tunable contrast [26]. On the other hand, an all-glass LPWG can give an ultra-low temperature sensitivity $(\sim 1$ $\mathrm{pm} /{ }^{\circ} \mathrm{C}$ ) [17]. Polymer LPWGs having a low temperature sensitivity have also been demonstrated with carefully designed waveguides $[19,20,23,28]$ and the condition for obtaining zero temperature sensitivity has been investigated both theoretically and experimentally [28].

The flexibility in the choice of waveguide materials and structures has also led to different methods for grating fabrication. Permanent gratings have been fabricated by the conventional photolithographic and etching technique [9$12,14,18,21,25,28,29]$, the nano-imprinting technique [22], and the UV/laser-writing technique [13,15,24]. Gratings with a tunable contrast based on the thermo-optic effect of polymer have been demonstrated by depositing suitable electrode gratings on polymer waveguides $[19,20,23,26,27]$.

In order to generate more sophisticated transmission characteristics, the idea of post-processing the profile of the waveguide cladding along a uniform LPWG has been suggested [32]. In practice, the waveguide cladding can be trimmed by using the conventional etching technique [33] or the UV/laser-irradiation technique [25,34]. Like LPFGs, LPWGs are inherently band-rejection filters. Nevertheless, widely tunable bandpass filters [35] and add/drop multiplexers [36-38] have been demonstrated recently with composite and parallel LPWGs, respectively. These devices extend substantially the functionality of LPWGs.

In this paper, the basic theory of LPWGs is first introduced, where the formulation of the sensitivity characteristics of a LPWG is highlighted. The techniques for the fabrication of various kinds of LPWGs are next reviewed. The flexibility of the technology is then illustrated by describing the characteristics of a number of representative devices.

\section{BASIC THEORY}

The transmission characteristics of a uniform LPWG can be analyzed by the coupled-mode theory $[8,16]$. The resonance wavelength $\lambda_{0}$, at which the coupling between the guided mode and the $m$-th order cladding mode is strongest, is given by the phase-matching condition:

$$
\lambda_{0}=\left(N_{0}-N_{m}\right) \Lambda
$$

where $N_{0}$ and $N_{m}$ are the mode indices of the guided mode and the cladding mode, respectively, and $\Lambda$ is the grating pitch. The curve showing the dependence of $\lambda_{0}$ on $\Lambda$ is called the phase-matching curve. The transmission coefficient at the wavelength $\lambda$ is given by 


$$
T=1-\frac{\kappa^{2}}{\kappa^{2}+\frac{\delta^{2}}{4}} \sin ^{2} \sqrt{\kappa^{2}+\frac{\delta^{2}}{4}} L
$$

where $\delta=(2 \pi / \Lambda)\left(\lambda_{0}-\lambda\right) / \lambda$ is a detuning parameter (phase mismatch) that measures the deviation of the wavelength $\lambda$ from $\lambda_{0}, \kappa$ is the coupling coefficient that measures the grating strength, which is proportional to the spatial overlap in the grating region between the guided mode and the cladding mode, and $L$ is the length of the grating. At the resonance wavelength $\lambda_{0}, \delta$ is equal to zero and the contrast is highest (i.e., the transmission is zero) when $\kappa$ is equal to $(n+1) \pi / 2$, where $n=0,1,2, \ldots$. In most practical cases, $n=0$ is used because the side lobes in the transmission spectrum becomes more significant as $n$ increases [39].

The resonance wavelength of a rejection band is in general sensitive to a number of physical parameters, such as temperature, surrounding refractive index, waveguide dimensions, grating pitch, etc. The sensitivity of the resonance wavelength to any physical parameter $X$ can be derived from Eq. (1) as

$$
\frac{d \lambda_{0}}{d X}=\gamma S_{X}
$$

with

$$
\gamma=\left[1-\Lambda\left(\frac{d N_{0}}{d \lambda}-\frac{d N_{m}}{d \lambda}\right)\right]^{-1}
$$

and

$$
S_{X}=\Lambda \sum_{i}\left(\frac{\partial N_{0}}{\partial Y_{i}}-\frac{\partial N_{\mathrm{m}}}{\partial Y_{i}}\right) \frac{\partial Y_{i}}{\partial X}+\frac{\lambda_{0}}{\Lambda} \frac{d \Lambda}{d X}
$$

where $Y_{i}$ represent all the waveguide parameters (the core and cladding thicknesses and the refractive indices in different regions) that are possibly affected by $X$. It can be seen from Eq. (3) that the sensitivity of a LPWG to any physical parameter depends on the same factor $\gamma$, which is determined by the dispersion properties of the modes (evaluated at $\lambda_{0}$ ). Setting $X=\Lambda$ in Eq. (3) leads to

$$
d \lambda_{0} / d \Lambda=\gamma \lambda_{0} / \Lambda
$$

Therefore, the slope of the phase-matching curve, $d \lambda_{0} / d \Lambda$, is a direct measure of the modal dispersion factor $\gamma[16,40,41]$. The value of $\gamma$ can be positive or negative, or even equal to infinity at the turning points of the phase-matching curve. For a fiber grating, because the fiber cladding is thick, $\gamma= \pm \infty$ is possible only with high-order cladding modes [40,41]. For a waveguide grating, however, turning points can occur along the phase-matching curves for low-order cladding modes by choosing appropriate cladding or core dimensions $[14,16]$. This property of the LPWG can be explored for the realization of highly sensitive tunable filters or sensors.

As an example, the temperature sensitivity factor $S_{T}$, with the thermal expansion of the grating ignored, is given by [42]

$$
S_{T}=\Lambda\left[C_{\mathrm{co}}\left(\eta_{0 \mathrm{co}}-\eta_{m \mathrm{co}}\right)+C_{\mathrm{cl}}\left(\eta_{0 \mathrm{cl}}-\eta_{m \mathrm{cl}}\right)\right]
$$

where $C_{\mathrm{co}}$ and $C_{\mathrm{cl}}$ are the thermal-optic coefficients of the core and the cladding, respectively. $\eta_{0 \mathrm{co}}$ and $\eta_{0 \mathrm{cl}}$ denote the fractional powers of the guided mode in the core and the cladding, respectively, and $\eta_{m c o}$ and $\eta_{m c l}$ denote the fractional powers of the cladding mode in the core and the cladding, respectively. When the cladding is thick enough, the mode fields in the substrate become negligible and we have $\eta_{0 \mathrm{co}}+\eta_{0 \mathrm{cl}} \cong 1$ and $\eta_{m \mathrm{co}}+\eta_{m \mathrm{cl}} \cong 1$. Equation (7) is then rewritten as

$$
S_{T}=\Lambda\left[\left(C_{\mathrm{co}}-C_{\mathrm{cl}}\right)\left(\eta_{0 \mathrm{co}}-\eta_{m \mathrm{co}}\right)\right] .
$$

According to Eqs. (3) and (8), a large temperature sensitivity can be obtained by using core and cladding materials that have significantly different thermal-optic coefficients or by choosing the cladding mode to give a large modal dispersion parameter $[11,13,14]$. The condition for achieving zero temperature sensitivity has also been investigated in detail recently [28]. It is found that zero temperature sensitivity can be achieved by using core and cladding materials that have significantly different thermo-optic coefficients. 
The 3-dB bandwidth of a LPWG of length $L$ at $\kappa L \sim \pi / 2$ can be derived from Eq. (2) as [41]

$$
|\Delta \lambda|_{3 d B}= \begin{cases}|\gamma| \Lambda \frac{0.8 \lambda_{0}}{L} & \gamma \neq \pm \infty \\ \frac{1.26 \lambda_{0}}{(c L \Delta D)^{1 / 2}} & \gamma= \pm \infty\end{cases}
$$

with

$$
\Delta D=\left(-\frac{2 \pi c}{\lambda_{0}^{2}} \frac{d^{2} \beta_{0}}{d \omega^{2}}\right)-\left(-\frac{2 \pi c}{\lambda_{0}^{2}} \frac{d^{2} \beta_{m}}{d \omega^{2}}\right)
$$

where $c$ is the free-space velocity of light, $\omega$ is the angular optical frequency, $\beta_{0}$ and $\beta_{m}$ are the propagation constants of the guided mode and the cladding mode, respectively, and $\Delta D$ is the difference in the group dispersion between the guided mode and the cladding mode. From Eq. (8), unless $\gamma= \pm \infty$, the 3 -dB bandwidth of a LPWG is proportional to the factor $\gamma$, the grating pitch $\Lambda$, and the resonance wavelength $\lambda_{0}$, while it is inversely proportional to the grating length $L$. According to Eq. (9), ultra-broadband mode conversion can be achieved with $\gamma= \pm \infty$ as $\Delta D$ approaches zero.

\section{LPWG FABRICATION}

Being a mature microfabrication technique, photolithography together with reactive-ion etching (RIE) has been applied extensively to the fabrication of corrugation gratings in glass $[10,12]$, polymer $[9,11,14,18,21,25,28]$, and semiconductor [29] waveguides. This technique involves the patterning of a waveguide with a metal grating mask by photolithography and the etching of the patterned waveguide by RIE. The grating is usually formed on the surface of the core of the waveguide. The desired corrugation depth, which determines the grating strength, is obtained by a careful control of the etching rate and time. This technique is capable of producing highly uniform gratings as well as high-contrast and clean filter spectra. Low efficiency and high cost are the obvious disadvantages. More seriously, because the grating is made before the cladding is formed, this technique demands a precise control of the cladding characteristics, which can be difficult for some polymer materials. A nano-imprint lithographic technique has also been demonstrated for the fabrication of a LPWG in a rib waveguide [22]. Compared with photolithography and RIE, the imprinting technique is simpler and less costly, and can be further developed for mass production. However, this technique also requires a careful control of the material properties and does not allow in-situ monitoring of the filter spectrum.

A LPWG has also been fabricated in a glass channel waveguide by a double ion-exchange process [17]. The first ionexchange process is used for cladding formation and the second ion-exchange process is used for forming a core with a periodically varying width by using a mask. Obviously, the same idea can be applied to the fabrication of LPWGs with other materials by using suitable lithographic techniques. The advantage of designing a grating in the form of a varying core width, instead of a varying core height, is that the etching depth on one or both sides of the core is defined by the mask rather than the conditions of the etching process, which should lead to more repeatable results.

To take advantage of the fact that polymers are in general UV sensitive, a UV-writing technique similar to that for the fabrication of LPFGs has been developed for the fabrication of LPWGs on polymer waveguides [15]. By exposing a waveguide with a photosensitive cladding to an excimer laser emitting at $248 \mathrm{~nm}$ through an amplitude mask, a grating can be formed in the cladding [15]. Because the cladding of a LPWG is usually quite thin (several micrometers), a grating in the cladding can still produce strong grating effects. A grating written into a photosensitive polymer core with the UV light through a non-photosensitive cladding has also been demonstrated [24]. The UV-writing technique allows real-time monitoring of the growth of the transmission spectrum of the grating. It also provides an effective means for post-processing the transmission characteristics of the grating [25]. It should be understood that the irradiation wavelength is not limited to $248 \mathrm{~nm}$. Any wavelength suitable for direct writing of polymer waveguide devices (including visible wavelengths) can be employed to implement this technique.

All the techniques described above produce permanent gratings that do not allow the contrast at the resonance wavelength to be controlled. An effective way to form a grating with a controllable strength is by depositing a periodic heater electrode on a polymer waveguide [19,20,23,26,27]. The grating is induced thermo-optically in the cladding by applying electric power to the electrode. The strength of the grating is thus controlled by the applied electric power. A 
LPWG filter with two sets of grating electrodes stacked up vertically [23] has been demonstrated, which offers an additional degree of freedom in the control of the transmission spectrum, compared with other similar devices $[19,20]$. Most recently, by using a different waveguide design and different polymer materials, a more power-efficient filter with wide tunability in both the center wavelength and the contrast has been demonstrated [26]. It is envisaged that nonuniform gratings and composite gratings can be induced thermo-optically in the same manner.

The central task in the design of a LPWG is to determine its pitch so that the rejection band can appear at the desired wavelength. Because the core-cladding index contrast of a waveguide is usually larger than that of a fiber, the pitch of a LPWG is usually several times shorter than that of a typical LPFG and, according to Eq. (9), a LPWG can be made shorter to give the same bandwidth. Most of the LPWGs demonstrated have a pitch varying from a few tens to $\sim 100$ micrometers and a length of $\sim 10 \mathrm{~mm}$. With an index contrast of the order of 0.01 (for typical polymer and glass waveguides), according to Eq. (1), the pitch required does not normally exceed $100-200 \mu \mathrm{m}$. In fact, a pitch as short as $11.8 \mu \mathrm{m}$ has been demonstrated with a semiconductor LPWG having a high index contrast [29]. The rejection bands observed with a long-pitch $(\sim 400 \mu \mathrm{m})$ grating in a high-index-contrast $(\sim 0.1)$ waveguide $[43,44]$ are dubious.

\section{EXAMPLES OF WIDELY TUNABLE LPWG FILTERS}

\subsection{Band-rejection filters}

Compared with LPFG filters, polymer-based LPWG filters can offer a much wider tuning range and higher tuning sensitivity. The resonance wavelength of a typical polymer LPWG can be tuned over the entire (C+L)-band from 1520 to $1610 \mathrm{~nm}$ with a temperature control of only $10-30{ }^{\circ} \mathrm{C}$ [9-15]. The temperature sensitivity of the resonance wavelength of a LPWG formed in a slab or ridge waveguide depends critically on the thickness of the waveguide cladding [12,13], so cladding thickness is an effective parameter for the control of the thermal tunability of a LPWG. On the other hand, the temperature sensitivity of a LPWG formed in a channel waveguide is much more sensitive to the core dimensions than the cladding thickness [14].

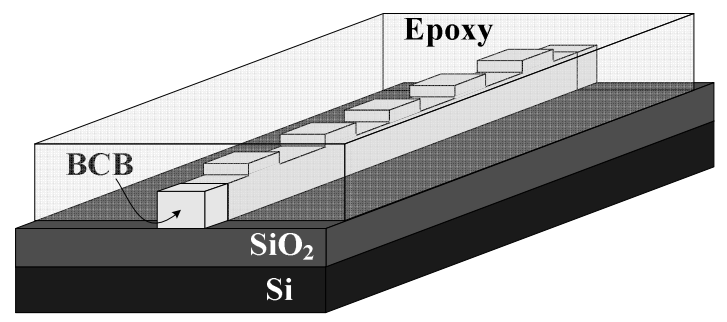

Fig. 1. Schematic diagram of a LPWG formed in an epoxy-clad BCB channel waveguide.

As an example, a uniform LPWG fabricated in a polymer channel waveguide is shown schematically in Fig. 1, where benzocyclobutene (BCB) and epoxy OPTOCAST 3505 are used as the core and the cladding material, respectively. Figure 2(a) compares the temperature sensitivities of two LPWG samples fabricated in such a waveguide structure (Sample A and Sample B) [14]. Both gratings have the same core width $(4.4 \mu \mathrm{m})$ and a similar cladding thickness $(\sim 7$ $\mu \mathrm{m})$, but Sample A has a core thickness of $2.0 \mu \mathrm{m}$ and Sample B has a core thickness of $4.5 \mu \mathrm{m}$. As shown in Fig. 2(a), Sample A exhibits a positive temperature sensitivity $\left(4.5\right.$ and $4.8 \mathrm{~nm} /{ }^{\circ} \mathrm{C}$ for the TE and TM polarizations, respectively), whereas Sample B a negative temperature sensitivity $\left(-5.3 \mathrm{~nm} /{ }^{\circ} \mathrm{C}\right.$ and $-3.1 \mathrm{~nm} /{ }^{\circ} \mathrm{C}$ for the TE and $\mathrm{TM}$ polarizations, respectively). That the temperature sensitivity changes sign is due to the sign change of the modal dispersion factor $\gamma$ as the core thickness increases. Therefore, the temperature sensitivity of a LPWG in a channel waveguide can be controlled over a wide range by simply controlling the core size through changing the modal dispersion factor [14]. The transmission spectra of Sample A for the TE polarization measured at different temperatures are shown in Fig. 2(b). The rejection band is clean and the contrast at the center wavelength varies from $20-30 \mathrm{~dB}$ over the temperature range shown in the figure. In general, the transmission characteristics of a LPWG are highly polarization sensitive, as shown by the results in Fig. 2(a). Nevertheless, a polarization-insensitive resonance wavelength has been demonstrated over a wide temperature range with a channel waveguide [11] and at a fixed temperature with a ridge waveguide [13]. 

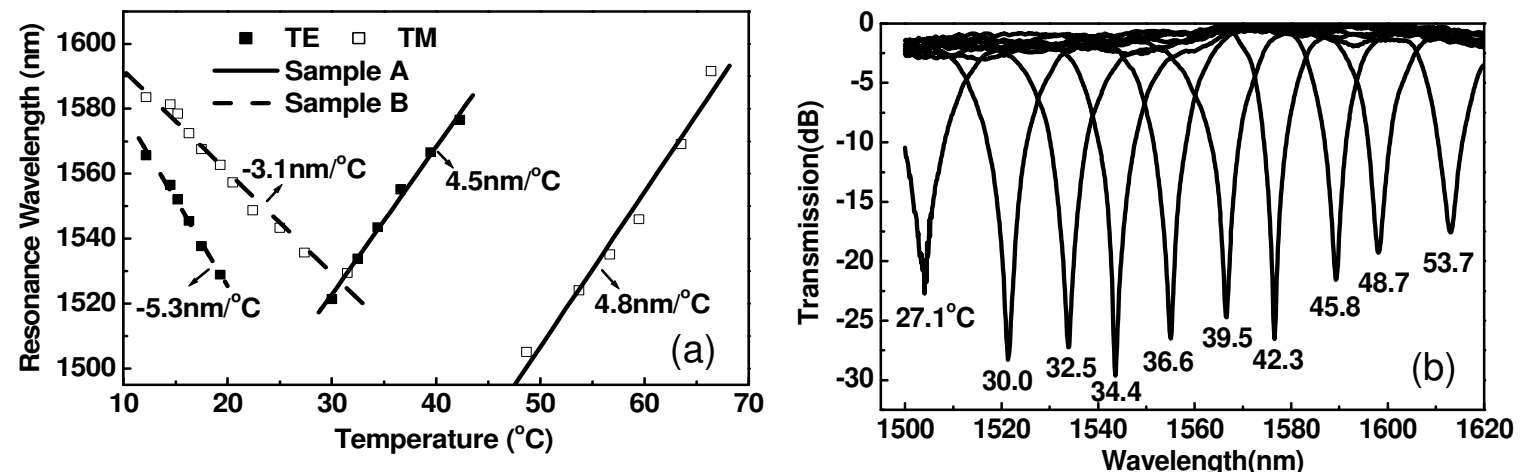

Fig. 2. (a) Measured temperature dependence of the resonance wavelength for Sample A and Sample B. (b) Normalized transmission spectra for the TE polarization measured at different temperatures for Sample A [14].

To generate more complicated transmission spectra in a controllable manner from a uniform LPWG, a UV postprocessing technique can be employed [25,34]. For example, a simple setup to realize a phase-shifted grating by the UVirradiation technique is shown in Fig. 3 with the results shown in Fig. 4, where the change of the transmission spectrum with the UV dosage ( $248 \mathrm{~nm}$ pulses from a $\mathrm{KrF}$ excimer laser) for an epoxy-clad $\mathrm{BCB}$ waveguide with a core size of 4.7 $\times 2.0 \mu \mathrm{m}^{2}$ and a cladding thickness of $6.3 \mu \mathrm{m}$ is monitored [25]. The temperature sensitivity of the resultant spectrum is $\sim 5 \mathrm{~nm} /{ }^{\circ} \mathrm{C}$. More sophisticated spectra can be generated in a similar way [25,34]. Non-uniformity can also be produced by etching the cladding along a uniform grating with the RIE process [33].

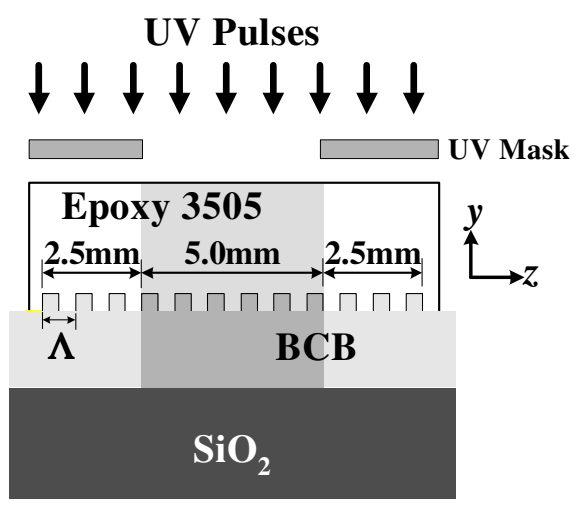

Fig. 3. UV-irradiation of a uniform LPWG through a mask for the generation of a controllable phase shift in the grating.
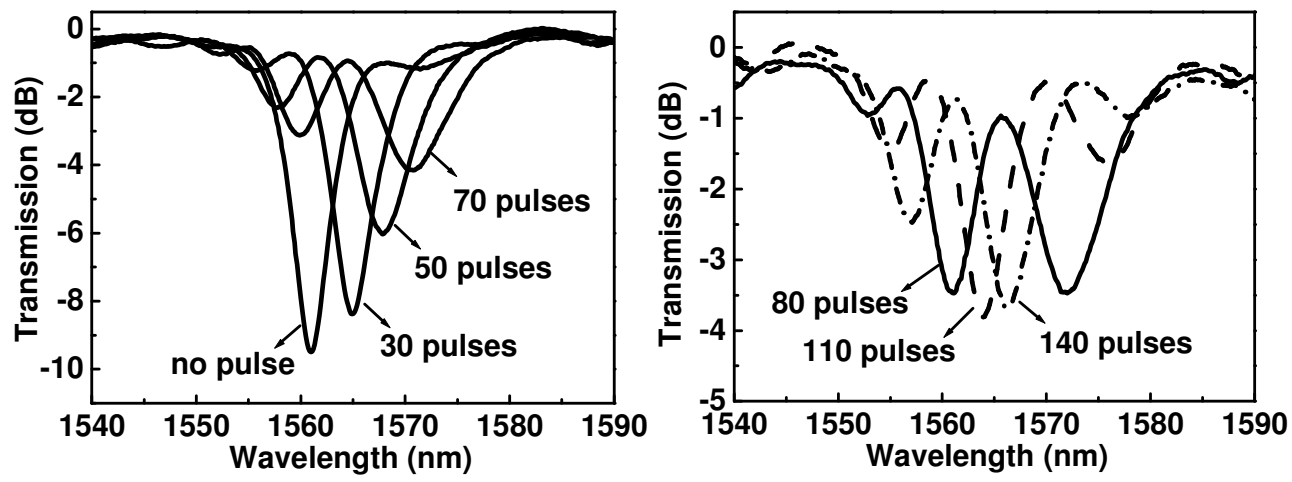

Fig. 4. Normalized transmission spectra of the UV-exposed LPWG sample measured at different UV doses [25]. 


\subsection{Polarization-dependent loss (PDL) compensators and variable attenuators}

A widely tunable LPWG filter with a polarization-sensitive rejection band can operate effectively as a polarizationdependent loss (PDL) compensator. By tuning the center wavelength of the rejection band of the filter, either the TE or TM light can be attenuated to the desired level at a particular wavelength. Although PDL compensation cannot be achieved simultaneously over a broad band, a wide wavelength-tuning range can ensure effective PDL compensation at any single wavelength within a broad band. For PDL compensation, the contrast at the center wavelength of the rejection band does not need to be large (a few dBs should be sufficient for most applications), but a large bandwidth is preferred.

To operate a LPWG as a variable attenuator, the contrast of the rejection band must be adjustable. Several polymerbased thermo-optic LPWGs have been demonstrated for that purpose, which differ in the material and the waveguide structure used and the way the periodic heater electrode is placed [19,20,23,26,27]. By using a buffer layer to isolate optically and mechanically the electrode from the waveguide cladding, a thermo-optic grating is formed when electric power is applied to the electrode $[19,20,23,27]$. With such a configuration, the contrast at the resonance wavelength increases with the applied electric power. For one device, the power required for changing the contrast by $27 \mathrm{~dB}$ is 851 $\mathrm{mW}[19,20]$. With the help of buffer layers, two sets of periodic heater electrodes can be stacked up vertically to produce more complicated transmission spectra [23]. Because these devices use thickly-clad channel waveguides and thermooptically matched core and cladding materials, their operating wavelengths have a low temperature sensitivity $(\sim 0.15$ $\mathrm{nm} /{ }^{\circ} \mathrm{C}$ ) and, therefore, cannot be tuned effectively. The use of a different waveguide structure together with different materials can solve this problem [26].

Figure 5 shows a metal grating (a thin periodic heater electrode made of chromium) deposited directly on the surface of the epoxy cladding of a BCB channel waveguide [26]. Because no buffer layer is used between the electrode and the cladding, even when no electric power is applied to the electrode, the metal grating itself produces a strong rejection band. When electric power is applied to the electrode, an additional thermo-optic grating is induced in the waveguide and thus changes the contrast of the rejection band. The waveguide structure together with the material system guarantees that the center wavelength of the rejection band can be tuned over a wide range. To achieve effective wavelength tuning, a temperature controller (e.g., a heat pump) is incorporated into the device to change the global temperature of the device.

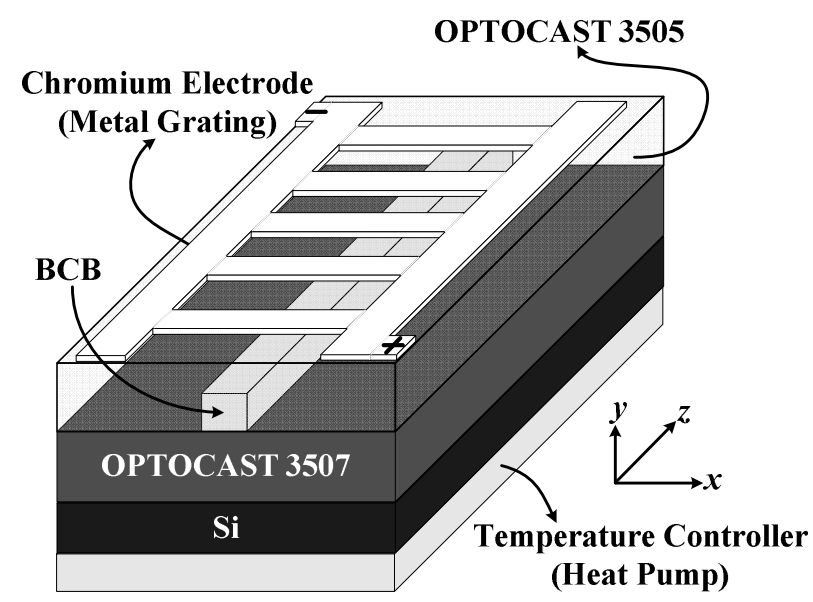

Fig. 5. Schematic diagram of a metal LPG formed in an epoxy-clad BCB channel waveguide.

The results for such a LPWG [26] are described below briefly. Figure 6(a) shows the normalized transmission spectra of the device for both polarizations measured at $21.6{ }^{\circ} \mathrm{C}$ when no current is applied to the electrode. The contrasts at the center wavelengths for the TE and TM polarizations are $29 \mathrm{~dB}$ and $26 \mathrm{~dB}$, respectively. As the chromium electrode is in direct contact with the cladding, it is a perturbation to the waveguide structure and thus acts very much like a corrugation grating. In practice, during the formation of the periodic electrode, stress is created periodically along the waveguide, which also acts as a grating. The transmission spectra shown in the figure should be the results of these grating effects. Figure 6(b) shows the temperature dependence of the center wavelength measured when no current is applied to the electrode. The center wavelengths for both polarizations increase with the temperature with a sensitivity of $\sim 2 \mathrm{~nm} /{ }^{\circ} \mathrm{C}$, 
which is an order of magnitude higher, compared with the other design $[19,20]$. When electric power is applied to the electrode, a thermo-optic grating is generated. Figure 7(a) shows the transmission spectra for the TE polarization measured at $20^{\circ} \mathrm{C}$ for different electric powers applied to the electrode. The contrast of the rejection band decreases from $34 \mathrm{~dB}$ to $6 \mathrm{~dB}$ when the power increases from 0 to $132 \mathrm{~mW}$. The electrode is actually deposited on top of three waveguides, so the average electric power required for each grating to achieve such a large change in contrast is only 45 $\mathrm{mW}$, which is almost 20 times smaller than that required in the other design [19,20]. The response time of the filter is also shorter. The lower power consumption and the faster response of the filter are the results of placing the electrode directly on the cladding surface without using any buffer layer. As shown in Fig. 7(a), the resonance wavelength shifts to the longer wavelength as the electric power increases. The reason is that the applied power not only induces a grating along the waveguide, but also raises the overall temperature of the device. To compensate for this effect, the temperature of the device can be varied with the heat pump to shift the wavelength to the desired value. Figure 7(b) shows how the contrast at the center wavelength decreases as the power increases with the center wavelength fixed at $\sim 1548 \mathrm{~nm}$ by an appropriate temperature control.
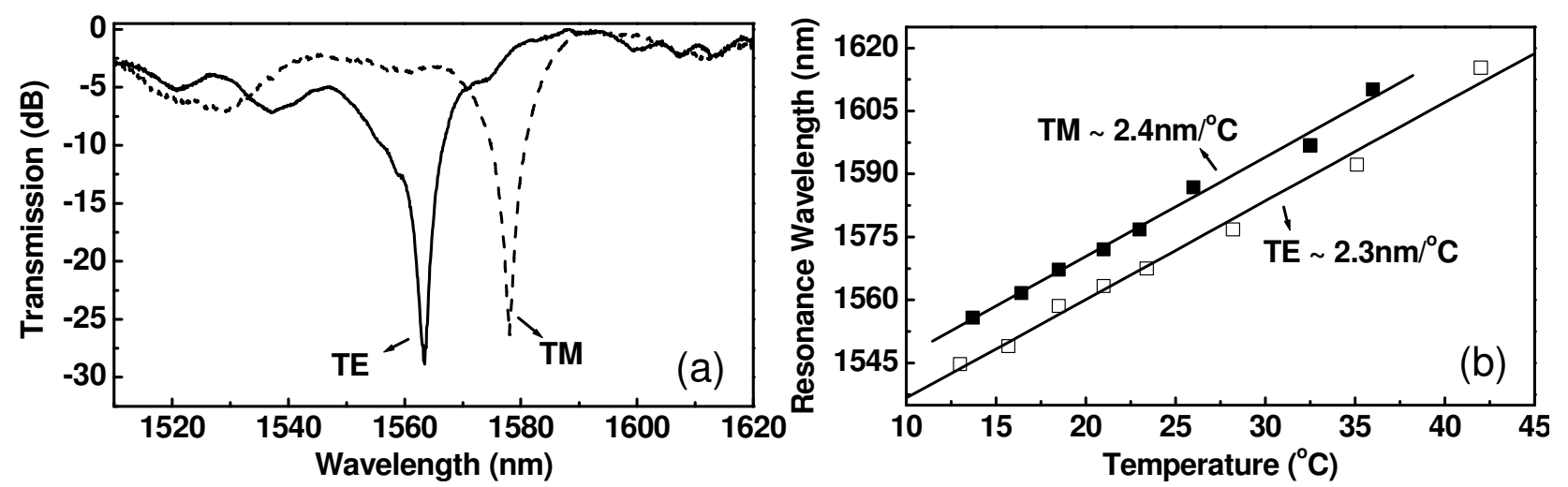

Fig. 6. (a) Normalized transmission spectra for the TE and TM polarizations measured at $21.6^{\circ} \mathrm{C}$ when no current was applied to the electrode. (b) Measured center wavelength as a function of the temperature. (Ref. [26])
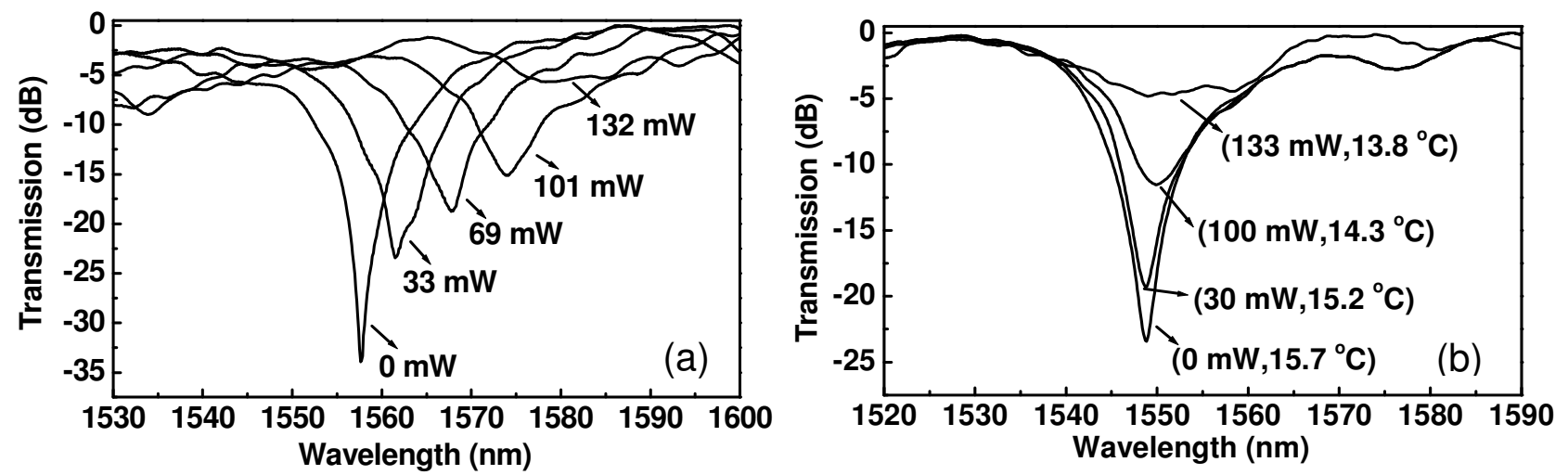

Fig. 7. (a) Normalized transmission spectra for the TE polarization measured at $20.0{ }^{\circ} \mathrm{C}$ as the applied power increases from 0 to 132 $\mathrm{mW}$. (b) Normalized transmission spectra measured at different temperatures and applied powers for the TE polarization. (Ref. [26])

\subsection{Bandpass filters}

Bandpass filters are required in many applications that involve the separation of wavelength channels. An attempt has been made to turn a LPWG into a bandpass filter [35]. The bandpass filter is shown schematically in Fig. 8, where two identical gratings with the same pitch $\Lambda$ and length $L$ are formed on the surface of the core of a single-mode channel waveguide and separated by a gap with a distance $d$. The gap between the two gratings, where the core is removed, serves to block the light that is not coupled to the cladding in the first grating from passing through the core and, at the 
same time, allows the light that is coupled to the cladding by the first grating to propagate to the second grating. In other words, the spectrum around the resonance wavelength of the grating is coupled to the cladding by the first grating and then collected from the second grating as the output, while the spectrum off the resonance wavelength is diffracted away when crossing the gap.

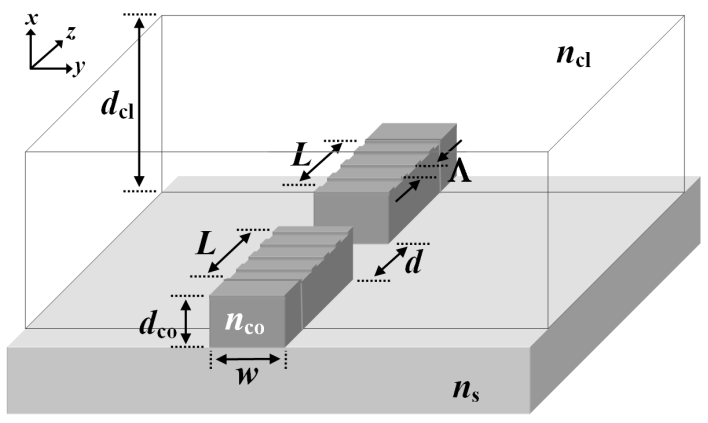

Fig. 8. Schematic diagram of a LPWG bandpass filter fabricated in an epoxy-clad BCB channel waveguide [35].
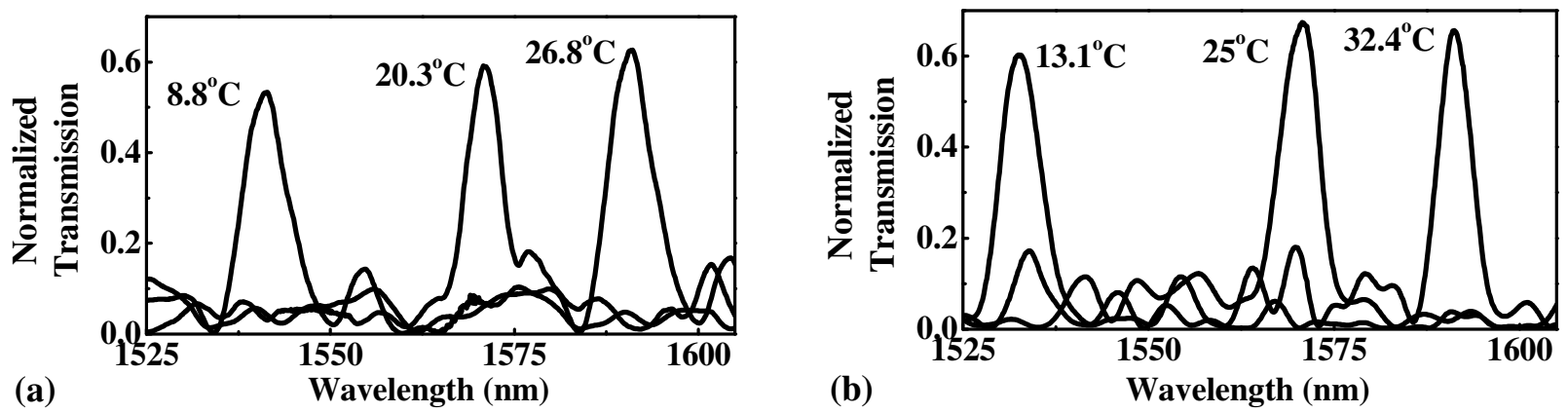

Fig. 9. Normalized transmission spectra of a fabricated LPWG bandpass filter measured at different temperatures for (a) the TE polarization and (b) the TM polarization [35].

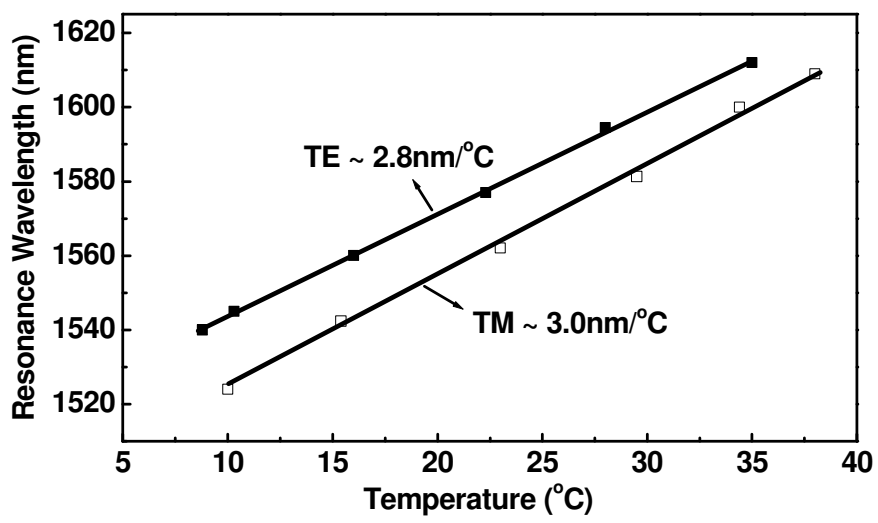

Fig. 10. Temperature dependence of the center wavelength of the pass-band of the filter. [35].

A number of such bandpass filters have been fabricated with epoxy-clad BCB channel waveguides using a grating pitch of $82 \mu \mathrm{m}$ and a gap distance of $500 \mu \mathrm{m}$ [35]. A set of experimental results is presented in Fig. 9, which shows clear bandpass characteristics. The transmission at the center wavelength of the pass-band is approximately $60 \%$. The FWHM (full-width-at-half-maximum) bandwidth of the passing band is about $6 \mathrm{~nm}$. Figure 10 shows the temperature 
dependence of the center wavelength of the pass-band of the filter. The temperature sensitivities for the TE and TM polarizations are 2.8 and $3.0 \mathrm{~nm} /{ }^{\circ} \mathrm{C}$, respectively, which allows the pass-band to be tuned over the $(\mathrm{C}+\mathrm{L})$-band with a temperature control of only $\sim 30^{\circ} \mathrm{C}$.

\subsection{Couplers and add/drop multiplexers}

Effective light coupling between two optical fibers by means of two parallel identical LPFGs has been analyzed in detail and demonstrated experimentally [45,46]. The outputs from the two fibers are complementary to each other, one showing band-rejection characteristics and the other showing band-pass characteristics. The structure of two parallel LPFGs thus operates as a broadband add/drop multiplexer. A six-port add/drop multiplexer using three parallel LPFGs has also been demonstrated recently [47]. The idea of realizing more robust add/drop multiplexers with parallel LPWGs formed on the same substrate has been proposed [32,36-38]. It is possible to achieve effective power transfer between parallel LPWGs over a distance of several millimeters. Furthermore, one can take advantage of the large temperature sensitivity of polymer LPWGs to achieve effective wavelength tuning.

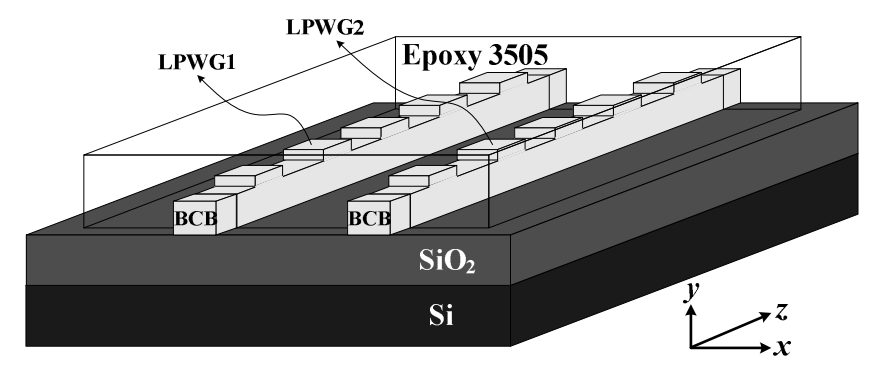

Fig. 11. Schematic diagram of a coupler formed with two parallel identical LPWGs in epoxy-clad BCB channel waveguides.

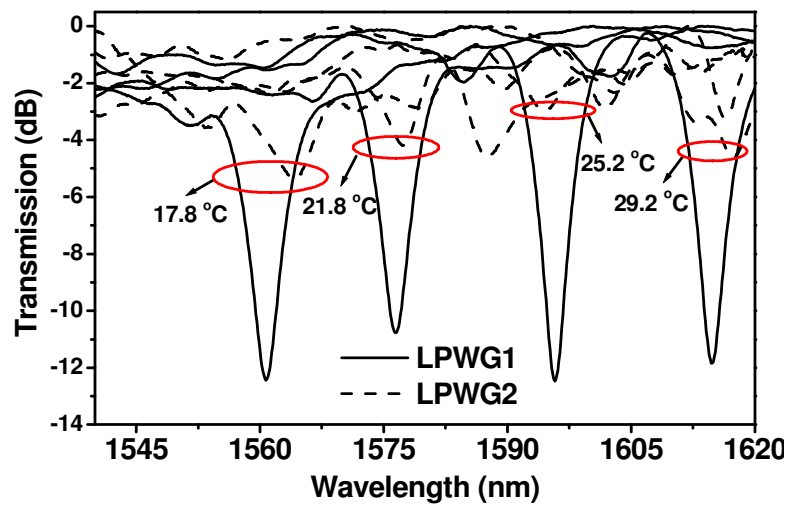

Fig. 12. Normalized transmission spectra measured from the launching core for the TE polarization at different temperatures when light is launched into one of the two cores (LPWG1 or LPWG2 in Fig. 11) of a fabricated coupler [38].

Figure 11 shows a coupler that consists of two identical LPGs formed in two parallel single-mode channel waveguides. The operation principle of the LPWG coupler is different from that of a LPFG coupler. In a LPFG coupler, power transfer between the two fibers is through evanescent-field coupling between the cladding modes of the two fibers $[45,46]$. In the LPWG coupler, light launched into one core is coupled to the cladding mode of the entire structure by the grating on the launching core, so the output from the launching core shows band-rejection characteristics. At the same time, light is coupled from the cladding mode of the entire structure to the other core by the other grating. The output from the coupled core thus shows band-pass characteristics. When the two cores are sufficiently separated, evanescentfield coupling between the two cores can be ignored. Unlike a LPFG coupler, there is no evanescent-field coupling in the LPWG coupler. Under appropriate conditions, $100 \%$ power exchange between the two waveguides can be achieved [36]. Effective coupling can also be achieved in an array of parallel LPWGs [37]. 
A number of LPWG couplers based on two parallel LPWGs have been fabricated with epoxy-clad BCB channel waveguides [38]. The BCB core has a dimension of $3.0 \times 1.8 \mu \mathrm{m}^{2}$ and a cladding thickness of $7 \mu \mathrm{m}$. The separation of the two cores is $8 \mu \mathrm{m}$. The two gratings have a pitch of $107 \mu \mathrm{m}$ and a length of $11 \mathrm{~mm}$ and are formed on the surface of the cores by the RIE process. Figure 12 shows the transmission spectra measured from the launching core when light is launched into one of the cores (LPWG1 or LPWG2 in Fig. 11). Results are given for the TE polarization at different temperatures. As shown in Fig. 12, the two gratings have close resonance wavelengths but significantly different contrasts at the resonance wavelengths. The difference is believed to be due to the fact that the two cores are not located symmetrically within the rectangular cladding, so the strengths of the two gratings, which depend on the spatial overlaps of the cladding mode of the entire structure and the guided modes of the individual cores, are different [38].

Figure 13 shows the transmission spectra measured from the coupled core when light is launched into one of the cores. Band-pass characteristics are clearly seen. When light is launched into LPWG1 and collected from LPWG2, as shown in Fig. 13(a), the maximum coupling efficiency is $\sim 60 \%$ at $25.2^{\circ} \mathrm{C}$. On the other hand, when light is launched into LPWG2 and collected from LPWG1, the maximum coupling coefficient is only $26 \%$ at $25.2{ }^{\circ} \mathrm{C}$, as shown in Fig. 13 (b). The difference in the coupling efficiency is another indication of the asymmetric behavior of the coupler caused by the slight asymmetry in the fabricated waveguide structure.
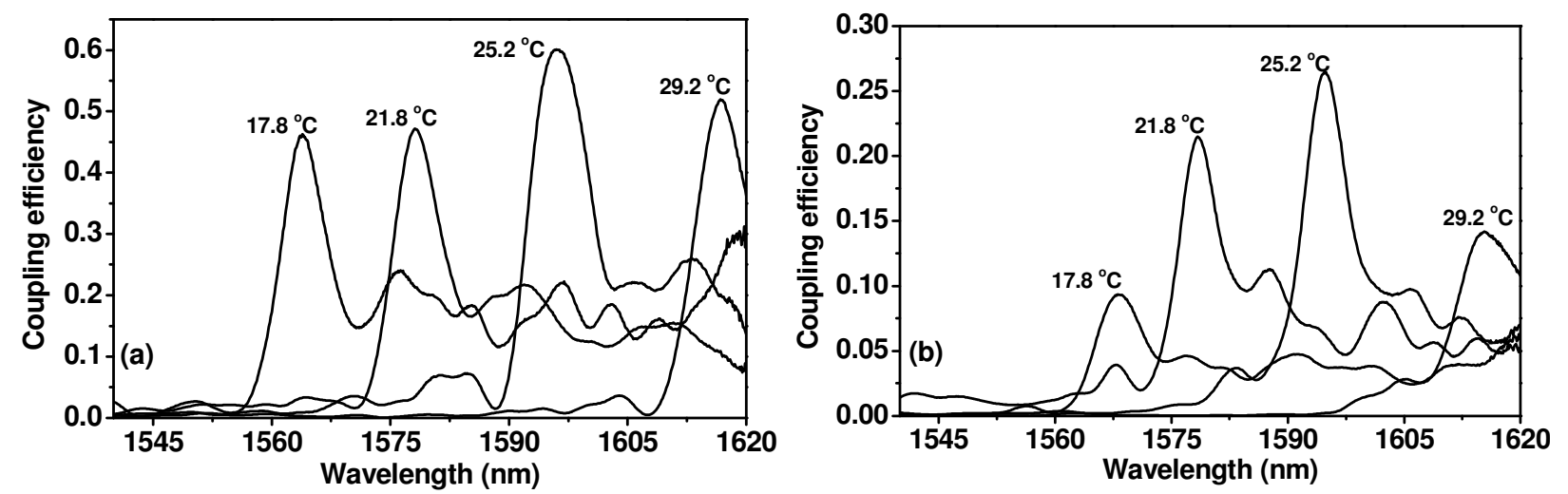

Fig. 13. Normalized transmission spectra measured from the coupled core for the TE polarization at different temperatures when light is launched into (a) LPWG1 or (b) LPWG2 of the coupler [38].

The temperature dependence of the resonance wavelength is shown in Fig. 14. The temperature sensitivity is $\sim 4.7$ $\mathrm{nm} /{ }^{\circ} \mathrm{C}$, which allows the operating wavelength of the coupler to be tuned over the $(\mathrm{C}+\mathrm{L})$-band with a temperature control of only $\sim 20^{\circ} \mathrm{C}$.

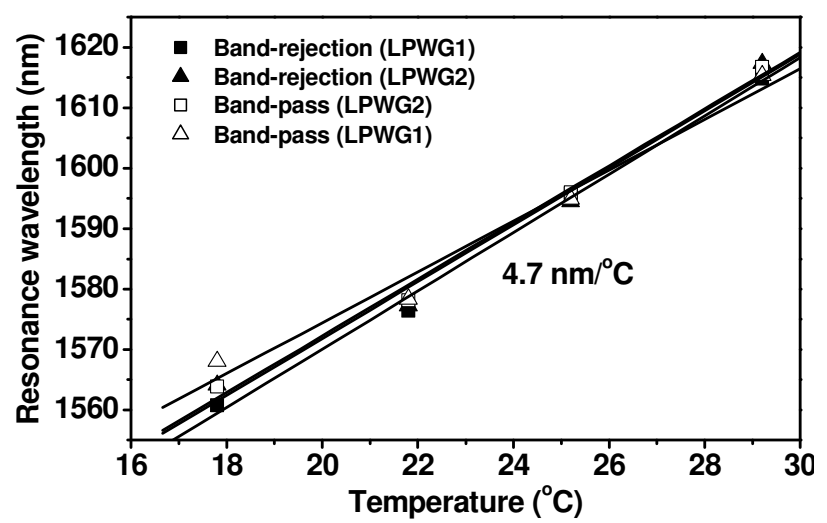

Fig. 14. Temperature dependence of the resonance wavelengths measured for LPWG1 and LPWG2 when light is launched into LPWG1 and LPWG2 of the coupler, respectively [38]. 
An array of ten parallel LPWGs with an ultra-large core separation of $80 \mu \mathrm{m}$ has also been demonstrated, which shows a maximum coupling efficiency of $\sim 11 \%$ between the two most strongly coupled cores and a temperature sensitivity of $-3.8 \mathrm{~nm} /{ }^{\circ} \mathrm{C}$ [38]. These preliminary results confirm the possibility of achieving strong light coupling between widely separated waveguides by using pure grating effects. Cladding uniformity is the main concern in the fabrication of such a large array of gratings with polymer waveguides.

\section{CONCLUSIONS}

This article provides an up-to-date review of the LPWG technology. Much progress has been made in this area of research since the subject was reviewed two years ago [32]. Clearly, the flexibility of the optical waveguide technology has been well appreciated, as there have been a large number of demonstrations of LPWGs with many different waveguide structures (slab, ridge, rib, and channel) using various materials (glass, polymer, and semiconductor). More importantly, the LPWG technology makes easier the realization of advanced devices with more sophisticated grating designs (non-uniform gratings, composite gratings, and coupled gratings), as illustrated by the examples in this article (phase-shifted gratings, band-pass filters, and add/drop multiplexers). There are practically unlimited possibilities in the design of new devices using LPWGs. All the tunable LPWG devices demonstrated so far rely on the large thermo-optic effect of polymer. There are good reasons to use polymer, as polymer is compatible with most optical materials and relatively easy to process, and shows great promise for mass production of components at a low cost. The large UVphotosensitivity of polymer has also been exploited to simplify the fabrication of LPWG devices. However, to achieve a much higher tuning/switching speed, the possibility of using electro-optic or piezo-electric material for the realization of LPWG devices should be explored. In fact, several reconfigurable filters based on electro-optically induced LPWGs have been analyzed [48-50]. Experimental demonstration is yet to be seen. Realization of dynamic LPWGs using liquid crystal or piezo-electric material like $\mathrm{ZnO}$ is a promising yet apparently untouched area. Most of the LPWGs demonstrated have a pitch in the range between a few tens to $\sim 100$ micrometers and a length of $\sim 10 \mathrm{~mm}$. These gratings are broadband devices that are most suited for coarse WDM applications. By using a large index contrast (as in a semiconductor structure), it should be possible to reduce the pitch to several micrometers or even shorter, in which case the grating can be made short enough (less than $1 \mathrm{~mm}$ ) for large-scale integration without broadening the bandwidth. On the other hand, when such a short-pitch LPWG is made long enough (several millimeters or longer), the bandwidth may be reduced to such an extent that the grating can be considered for applications even in dense WDM systems. It is clear to the author that there is a wide scope for further development of the LPWG technology and LPWG is evolving gradually into a versatile structure for the realization of new integrated optical devices with potential applications in optical communication and sensing.

\section{ACKNOWLEDGEMENTS}

The author wishes to thank his team members, Q. Liu, K. P. Lor, C. K. Chow, Y. Bai, and H. P. Chan, for their contributions to the studies presented in this paper, and Q. Liu specifically for his assistance in preparing this article. The research work for this article was supported by the Research Grants Council of the Hong Kong Special Administrative Region, China, under Projects CityU 1248/04E and CityU 112005.

\section{REFERENCES}

1. A. M. Vengsarkar, P. J. Lemaire, J. B. Judkins, V. Bhatia, T. Erdogan, and J. E. Sipe, "Long-period fiber gratings as band-rejection filters," J. Lightwave Technol., 14(1), 58-65 (1996).

2. K. S. Chiang and Q. Liu, "Long-period grating devices for application in optical communication," Proc. $5^{\text {th }}$ International Conference on Optical Communications and Networks (ICOCN 2006), Chongqing, China, (2006) (to appear).

3. S. W. James and R. P. Tatam, "Optical fibre long-period grating sensors: characteristics and application," Meas. Sci. Technol., 14(5), pp. R49-R61 (2003).

4. X. Shu, T. Allsop, B. Gwandu, L. Zhang, and I. Bennion, "High-temperature sensitivity of long-period gratings in B-Ge codoped fiber," IEEE Photon. Technol. Lett., 13(8), 818-820 (2001). 
5. A. A. Abramov, A. Hale, R. S. Windeler, and T. A. Strasser, "Widely tunable long-period fibre gratings," Electron. Lett., 35(1), 81-82 (1999).

6. K. S. Chiang, Y. Liu, M. N. Ng, and X. Dong, "Analysis of etched long-period fibre grating and its response to external refractive index," Electron. Lett., 36(11), 966-967 (2000).

7. V. Rastogi and K. S. Chiang, "Long-period grating in a four-layer planar waveguide", Proc. $5^{\text {th }}$ International Conference on Fiber Optics and Photonics (Photonics 2000), Calcutta, India, 19-21 (2000).

8. V. Rastogi and K. S. Chiang, "Long-period gratings in planar optical waveguides," Appl. Opt., 41(30), 6351-6355 (2002).

9. K. S. Chiang, C. K. Chow, K. P. Lor, H. P. Chan, V. Rastogi, and Y. M. Chu, "Long-period grating filters in benzocyclobutene (BCB) waveguides," Proc. Second Asia-Pacific Polymer Fibre Optics Workshop, Hong Kong, China, 81-83 (2003).

10. K. S. Chiang, K. P. Lor, C. K. Chow, H. P. Chan, V. Rastogi, and Y. M. Chu, "Widely tunable long-period gratings fabricated in polymer-clad ion-exchanged glass waveguides," IEEE Photon. Technol. Lett., 15(8), 1094-1096 (2003).

11. K. S. Chiang, C. K. Chow, H. P. Chan, Q. Liu, and K. P. Lor, "Widely tunable polymer long-period waveguide grating with polarization-insensitive resonance wavelength," Electron. Lett., 40(7), 422-423 (2004).

12. Y. M. Chu, Q. Liu, and K. S. Chiang, "Control of temperature sensitivity of long-Period waveguide grating by etching of cladding," Proc. $9^{\text {th }}$ Optoelectronics and Communications Conference (OECC 2004), Yokohama, Japan, 920-921 (2004).

13. Q. Liu, K. S. Chiang, and K. P. Lor, "Long-period gratings in polymer ridge waveguides," Opt. Express.,13(4), 1150-1160 (2005).

14. Q. Liu, K. S. Chiang, K. P. Lor and C. K. Chow, "Temperature sensitivity of a long-period waveguide grating in a channel waveguide," Appl. Phys. Lett., 86(24), 241115-1-241115-3 (2005).

15. K. P. Lor, Q. Liu, and K. S. Chiang, "UV-written long-period gratings on polymer waveguides," IEEE Photon. Technol. Lett., 17(3), 594-596 (2005).

16. Q. Liu, K. S. Chiang, and V. Rastogi, "Analysis of corrugated long-period gratings in slab waveguides and their polarization dependence," J. Lightwave Technol., 21(12), 3399-3405 (2003).

17. M. Christophe, H. Bertrand, C. Laurent, O. Jacquin, and G. Cyril, "Advanced spectral filtering functionalities in ionexchanged waveguides with artificial cladding gratings," Opt. Commun., 233(1-3), 97-106 (2004).

18. M. S. Kwon, K. H. Kim, Y. H. Oh, and S. Y. Shin, "Fabrication of an integrated optical filter using a large-core multimode waveguide vertically coupled to a single-mode waveguide," Opt. Express,11(18), 2211-2216 (2003).

19. M. S. Kwon and S. Y. Shin, "Tunable polymer waveguide notch filter using a thermooptic long-period grating," IEEE Photon. Technol. Lett., 17(1), 145-147 (2005).

20. M. S. Kwon and S. Y. Shin, "Characteristics of polymer waveguide notch filters using thermooptic long-period gratings," IEEE J. Sel. Top. Quantum Electron., 11(1), 190-196 (2005).

21. M. S. Kwon and S. Y. Shin, "Refractive index sensitivity measurement of a long-period waveguide grating," IEEE Photon. Technol. Lett., 17(9), 1923-1925 (2005).

22. A. Perentos, G. Kostovski, and A. Mitchell, "Polymer long-period raised rib waveguide gratings using nano-imprint lithography," IEEE Photon. Technol. Lett., 17(12), 2595-2597 (2005).

23. M. S. Kwon and S. Y. Shin, "Polymer waveguide notch filter using two stacked thermooptic long-period gratings," IEEE Photon. Technol. Lett., 17(4), $792-794$ (2005).

24. K. S. Chiang, K. P. Lor, and Q. Liu, "UV-written buried polymer long-period waveguide gratings," Proc. Bragg Gratings, Poling \& Photosensitivity 2005 (BGPP 2005), Sydney, Australia, 347-349 (2005).

25. Q. Liu, K. S. Chiang, and K. P. Lor, "Tailoring the transmission characteristics of polymer long-period waveguide gratings by UV irradiation," IEEE Photon. Technol. Lett., 17(11), 2340-2342 (2005).

26. K. S. Chiang, C. K. Chow, Q. Liu, H. P. Chan, and K. P. Lor, "Band-rejection filter with widely tunable center wavelength and contrast using metal long-period grating on polymer waveguide," IEEE Photon. Technol. Lett., 18(9), 1109-1111 (2006).

27. M. S. Kwon, Y. B. Cho, and S. Y. Shin, "Experimental demonstration of a long-period grating based on the sampling theorem," Appl. Phys. Lett., 88(22), 211103-1-211103-3 (2006).

28. Q. Liu, K. S. Chiang, and K. P. Lor, "Condition for the realization of a temperature-insensitive long-period waveguide grating," Opt. Lett., (2006) (to appear). 
29. H. Tabuchi, T. Abe, K. Terada, and K. Utaka, "Long-period-grating-loaded semiconductor separate-confinement heterostructure waveguide for polarization-independent gain-equalizing device," J. Appl. Phys., 44(49), L1488L1490 (2005).

30. M. S. Kwon and S. Y. Shin, "Tunable notch filter using a thermooptic long-period grating," J. Lightwave Technol., 22(8), 1968-1975 (2004).

31. M. S. Kwon and S. Y. Shin, "Theoretical investigation of a notch filter using a long-period grating based on the sampling theorem," Opt. Commun., 263(2), 214-218 (2006).

32. K. S. Chiang, K. P. Lor, Q. Liu, C. K. Chow, Y. M. Chu, and H. P. Chan, "Long-period waveguide gratings,” Jpn. J. Appl. Phys., 43(8B), 5690-5696 (2004).

33. M. S. Kwon and S. Y. Shin, "Spectral tailoring of uniform long-period waveguide grating by the cladding thickness control," Opt. Commun., 250(1-3), 41-47 (2005).

34. Q. Liu and K. S. Chiang, "Design of long-period waveguide grating filter by control of waveguide cladding profile," J. Lightwave Technol., 24(9), (2006) (to appear).

35. Y. M. Chu, K. S. Chiang, and Q. Liu, "Widely tunable optical bandpass filter by use of polymer long-period waveguide gratings," Appl. Opt., 45(12), 2755-2760 (2006).

36. Y. K. Bai and K. S. Chiang, "Analysis and design of long-period waveguide grating couplers", J. Lightwave Technol., 23(12), 4363-4373 (2005).

37. Y. K. Bai and K. S. Chiang, "Analysis of long-period waveguide grating arrays," J. Lightwave Technol., 24(10), (2006) (to appear).

38. Y. K. Bai, Q. Liu, K. P. Lor, and K. S. Chiang, "Widely tunable long-period waveguide grating couplers", Proc. $5^{\text {th }}$ International Conference on Optical Communications and Networks (ICOCN 2006), Chongqing, China, (2006) (to appear).

39. F. Y. M Chan and K. S. Chiang, "Analysis of apodized phase-shifted long-period fiber gratings", Opt. Commun., 244, 233-243 (2005).

40. T. W. MacDougall, S. Pilevar, C. W. Haggans, and M. A. Jackson, "Generalized expression for the growth of long period gratings," IEEE Photon. Technol. Lett., 10(10), 1449-1451 (1998).

41. X. Shu, L. Zhang, and I. Bennion, "Sensitivity characteristics of long-period fiber gratings," J. Lightwave Technol., 20(2), pp. 255-266 (2002).

42. M. N. Ng and K. S. Chiang, "Thermal effects on the transmission spectra of long-period fiber gratings," Opt. Commun., 208, 321-327 (2002).

43. H. C. Tsoi, W. H. Wong, and E. Y. B. Pun, "Polymeric long-period waveguide gratings," IEEE Photon. Technol. Lett., 15(5), 721-724 (2003).

44. H. Y. Tang, W. H. Wong, and E. Y. B. Pun, "Long-period polymer waveguide grating device with positive temperature sensitivity," Appl. Phys. B: Lasers Opt., 79(1), 95-98 (2004).

45. K. S. Chiang, Y. Liu, M. N. Ng, and S. Li, "Coupling between two parallel long-period fibre gratings," Electron. Lett., 36(16), 1408-1409 (2000).

46. K. S. Chiang, F. Y. M. Chan, and M. N. Ng, "Analysis of two parallel long-period fiber gratings," J. Lightwave Technol., 22(5), 1358-1366 (2004).

47. Y. Liu and K. S. Chiang, "Broadband optical coupler based on evanescent-field coupling between three parallel long-period fiber gratings," IEEE Photon. Technol. Lett., 18(1), 229-231 (2006).

48. M. Kulishov, X. Daxhelet, M. Gaidi, and M. Chaker, "Electronically reconfigurable superimposed waveguide longperiod gratings," J. Opt. Soc. Amer. A, 19(8), 1632-1648 (2002).

49. M. Kulishov and X. Daxhelet, "Reconfigurable $\pi$-shifted and Mach-Zehnder bandpass filters on the basis of electrooptically induced long-period gratings in a planar waveguide," J. Lightwave Technol., 21(3), 854-861 (2003).

50. M. Kulishov, X. Daxhelet, M. Gaidi, and M. Chaker, "Transmission spectrum reconfiguration in long-period gratings electrically induced in Pockels-type media with the help of a periodical electrode structure," J. Lightwave Technol., 22(3), 923-933 (2004). 